\title{
Multidisciplinary Management of Early-Stage Rectal Cancer
}

\author{
John G. Phillips, MD; ; Theodore S. Hong, $\mathrm{MD}^{\mathrm{b}}$; and David P. Ryan, MDc
}

\begin{abstract}
Because patients with locally advanced rectal cancer are at high risk for both recurrence and distant disease, they require adjuvant therapy. In the United States, the current standard of care is neoadjuvant chemoradiation followed by surgery and adjuvant chemotherapy. Neoadjuvant chemoradiation has been shown to improve local recurrence rates and decrease toxicity. However in the era of total mesorectal excision surgery, no study has shown a survival benefit to either chemoradiation or postoperative chemotherapy. Newer biologic therapies, although promising in initial early trials, have yet to show a significant benefit in adjuvant therapy for rectal cancer. (JNCCN 2012;10:1577-1585)
\end{abstract}

\section{NCCN: Continuing Education}

\section{Accreditation Statement}

This activity has been designated to meet the educational needs of physicians and nurses involved in the management of patients with cancer. There is no fee for this article. No commercial support was received for this article. The National Comprehensive Cancer Network (NCCN) is accredited by the ACCME to provide continuing medical education for physicians.
NCCN designates this journal-based CME activity for a maximum of 1.0 AMA PRA Category 1 Credit(s) ${ }^{\mathrm{TM}}$. Physicians should claim only the credit commensurate with the extent of their participation in the activity.

NCCN is accredited as a provider of continuing nursing education by the American Nurses Credentialing Center's Commission on Accreditation.

This activity is approved for 1.0 contact hour. Approval as a provider refers to recognition of educational activities only and does not imply ANCC Commission on Accreditation approval or endorsement of any product. Accredited status does not imply endorsement by the provider of the education activity (NCCN). Kristina M. Gregory, RN, MSN, OCN, is our nurse planner for this educational activity.

All clinicians completing this activity will be issued a certificate of participation. To participate in this journal CE activity: 1) review the learning objectives and author disclosures; 2) study the education content; 3 ) take the post-test with a $70 \%$ minimum passing score and complete the evaluation at http://education.nccn.org/ node/6277; and 4) view/print certificate.

Release date: December 1, 2012; Expiration date: December 1, 2013.

\section{Learning Objectives}

Upon completion of this activity, participants will be able to:

- Describe the current standard of care for the management of patients with rectal cancer.

- Discuss the role of adjuvant therapy in the management of rectal cancer.

\footnotetext{
From the aHarvard Radiation Oncology Program, Harvard Medical School, and the Departments of 'Radiation Oncology and 'Medicine, Massachusetts General Hospital, Boston, Massachusetts.

Submitted June 8, 2012; accepted for publication October 8, 2012

The authors (John G. Phillips, MD; Theodore S. Hong, MD; and David P. Ryan, MD) have disclosed that they have no financial interests, arrangements, affiliations, or commercial interests with the manufacturers of any products discussed in this article or their competitors.

Correspondence: David P. Ryan, MD, Massachusetts General Hospital Cancer Center, Yawkey 7E, 55 Fruit Street, Boston, MA 02114. E-mail: dpryan@partners.org
}

\section{EDITOR}

Kerrin M. Green, MA, Assistant Managing Editor, Journal of the National Comprehensive Cancer Network.

Ms. Green has disclosed that she has no relevant financial relationships.

\section{CE AUTHOR}

Nicole B. Fair, BS, Manager, Continuing Education and Grants Ms. Fair has disclosed that she has no relevant financial relationships.

Kristina M. Gregory, RN, MSN, OCN, Vice President, Clinical Information Operations

Ms. Gregory has disclosed that she has no relevant financial relationships. 
Patients with AJCC stage II (invasion through the muscularis layers) and III (node-positive) rectal cancer are at high risk for both locoregional and distant failure. Chemoradiation is used to lower the rate of local recurrence, and adjuvant chemotherapy is used to lower the rate of both systemic and local recurrence. The initial principles of adjuvant chemotherapy were established before the advent of total mesorectal excision (TME) and imaging that allows evaluation of the $\mathrm{T}$ stage and estimation of the circumferential margin. This article outlines the general principles established by the early randomized studies, reflects on these principles in the era of TME and pelvic imaging, and reviews new areas of investigation.

\section{Early Randomized Studies: What Was Learned?}

The initial early randomized studies are summarized in Table 1. The Gastrointestinal Study Group (GITSG) established that patients with locally advanced rectal cancer had better outcomes when both radiation and chemotherapy were used in the adjuvant setting, socalled combined modality therapy. ${ }^{1,2}$ Subsequently, the Mayo/North Central Cancer Treatment Group (NCCTG) 79-47-51 randomized 204 patients with locally advanced rectal cancer treated with surgery to either postoperative radiation to $50.4 \mathrm{~Gy}$ or the same radiation regimen with concurrent bolus 5 -FU with semustine both preceded and followed by adjuvant 5-FU and semustine. Five-year local recurrence rates were significantly better in the chemoradiation arm (41\% vs. $63 \%$ ), with a significant 5 -year overall survival (OS) benefit of $55 \%$ versus $40 \% .^{3}$ Further refinement in the chemoradiation regimen came from the joint Intergroup/NCCTG 86-47-51 trial, which showed a benefit in OS (70\% vs. $60 \%$ at 46 months median follow-up) for continuous-infusion 5-FU over traditional bolus 5-FU. ${ }^{4}$ Semustine was eliminated from the adjuvant chemotherapy regimen when an analysis of the initial 445 accrued patients showed a statistically insignificant early effect.

The first 2 NSABP studies, R-01 and R-02, added confusion to the management of locally advanced rectal cancer. From 1977 to 1986, NSABP R-01 randomized 555 patients to surgery alone, surgery followed by radiation therapy, or surgery followed by chemotherapy (5-FU, semustine, vincristine).
A statistically significant improvement in OS was seen for patients randomized to chemotherapy but not for those randomized to radiation therapy alone. Radiation therapy decreased the risk of locoregional recurrence from $25 \%$ to $16 \%(P=.06)$ but had no effect on survival. ${ }^{2}$ The NSABP R-02 study subsequently randomized 694 patients to adjuvant chemotherapy or combined modality therapy for patients with stage II and III rectal cancer and there was no difference in OS. Combined modality therapy did reduce locoregional recurrence from $13 \%$ to $8 \%(P=.02) .^{5}$ Despite the lack of survival benefit when comparing combined modality therapy with chemotherapy alone, and before TME, combined modality therapy became the standard of care because of the prior GITSG and NCCTG studies and its effect on reducing locoregional recurrence.

\section{Local Control in the Era of TME}

Perhaps the greatest advance in rectal cancer came through the development of TME as the oncologic surgery of choice for the management of rectal cancer. TME removes the intact mesorectal fascia layer surrounding the rectum through sharp dissection, and as a result removes the majority of draining lymph nodes. In addition, the technique allows for improved rate of $\mathrm{R} 0$ resection with regard to the circumferential radial margin. Heald et $\mathrm{al}^{6,7}$ first described TME at the Basingstoke District Hospital in the early 1980s. They reported on an initial series of 100 patients treated with TME and no adjuvant radiation in whom no local recurrences were seen during the first 2 years of follow-up. Subsequent follow-up of this initial series showed a $4 \%$ local recurrence rate at 5 years. ${ }^{8}$ Multiple surgical series were published showing similar results, such as the experience from Memorial Sloan-Kettering Cancer Center in which 246 patients were treated with TME. Patients with T3 disease and those with positive nodes had a local recurrence rate of $4 \%$ and $8.1 \%$, respectively, at 5 years. ${ }^{9}$ Later, Havenga et $\mathrm{al}^{10}$ published a retrospective analysis of 1141 prospectively collected patients from different centers that compared the locoregional recurrence rates for stages II and III rectal cancer. At centers where TME was practiced, locoregional recurrence rates were $4 \%$ to $8 \%$, compared with $32 \%$ to $35 \%$ in the centers using conventional abdominoperineal 
resections (APRs) or low-anterior resections (LARs). ${ }^{10}$ Based on these experiences, TME became the accepted standard in the field of rectal cancer surgery, although the ability to ensure quality control in studies lagged.

This dramatic decrease in locoregional failure raised questions about the need for radiation and chemotherapy. The Dutch Colorectal Cancer Trial (CKVO 95-04) attempted to determine the benefit of radiation therapy by randomizing patients to a short course of preoperative radiation (25 Gy in 5 fractions) followed by TME or TME alone. They found no difference in survival between the arms, but local recurrence was significantly better in the radiation arm $(2.4 \%$ vs. $8.2 \% ; P<.001)$. This significant benefit in local control persisted with longer follow-up: $5 \%$ versus $11 \%$ at 10 years $(P<.0001) .{ }^{11,12}$ On subset analysis, for patients with TNM stage III cancer with a negative circumferential resection margin, 10-year survival was $50 \%$ in the preoperative radiotherapy group versus $40 \%$ in the surgery-alone group $(P=.032)$. The Dutch Colorectal Cancer Trial served to move the field in 3 ways. First, it established that TME is the standard of care, because local control rates of $90 \%$ had not been seen in previous multicenter randomized trials for stage II and III rectal cancer treated with surgery alone. Second, it established that radiation was useful even in the setting of TME. Finally, the poor $\mathrm{OS}$ at 10 years highlighted the importance of adjuvant chemotherapy, which was omitted as was the standard custom at the time in the Netherlands.

\section{Neoadjuvant Chemoradiation}

Occurring at the same time as the movement towards TME, a series of trials attempted to address feasibility and outcomes of neoadjuvant chemoradiation. In many of the early trials, patients had difficulty completing chemoradiation or chemotherapy after surgery because of toxicity. Postoperatively, loops of small bowel will fill the lower pelvis and be subject to radiation, thus contributing to increased toxicity. In addition, surgeons hoped that preoperative therapy could make patients who needed an APR eligible for a sphincter-sparing LAR. The argument against preoperative therapy centered around the inability to accurately assess T3 versus T1/2 disease on staging CT scans. ${ }^{13}$ With the advent of endorectal ultrasound and MRI, the accuracy of predicting T3 disease improved dramatically to $90 \%$ in many series. ${ }^{14-17}$ The first attempt to perform a randomized controlled trial was by the NSABP. NSABP R-03 randomized 267 patients with locally advanced rectal cancer to either pre- or postoperative chemoradiation using 5-FU and leucovorin and 50.4 Gy of radiation accomplished in 28 fractions. This trial closed early because of poor accrual. Analysis of the accrued patients showed a 5-year disease-free survival (DFS) improvement in the preoperative chemoradiation arm $(64.7 \%$ vs. $53.4 \%)$ and a trend toward a 5 -year OS benefit. ${ }^{18}$ No difference was seen in locoregional recurrence. Notably, this trial did not require that patients undergo a TME and no difference was seen in conversion to LAR.

The debate between the advocates of preoperative versus postoperative therapy continued until the CAO/ARO/AIO-94 German Rectal Study, which randomized 823 patients with locally advanced rectal cancer to either preoperative chemoradiation to 50.4 Gy in 28 fractions with concurrent continuous infusion 5-FU, or postoperative chemoradiation to 55.8 Gy with continuous infusion 5-FU. TME was prospectively mandated in this trial, and both groups received 4 cycles of bolus 5-FU after all other therapies. ${ }^{19,20}$ No difference was seen in OS, DFS, or distant metastases at 5 or 10 years. A significant reduction in local recurrence was seen in the preoperative arm at 5 years $(6 \%$ vs. $13 \%)$, which persisted at 10 years $(7.1 \%$ vs. $10.1 \%)$. Fewer acute and late toxicities and an $8 \%$ pathologic complete response rate (pCR) were also seen. They also reported improved sphincter-sparing surgeries in the preoperative arm in patients deemed to require APR at initial clinical staging (39\% vs. 19\%). Notably, 18\% of patients assigned to immediate surgery were incorrectly overstaged as having T3 disease according to endorectal ultrasound. Nevertheless, this study established preoperative chemoradiation as the standard of care in patients with locally advanced rectal cancer.

\section{Neoadjuvant Chemoradiation Versus Radiation Alone}

Although the role of chemotherapy was clearly established in the context of postoperative radiation therapy, early studies of preoperative radiation did not address the issue of concurrent chemotherapy. ${ }^{12}$ The role of concurrent chemotherapy was questioned 
Phillips et al

because it seemed that radiation alone, as given in the Dutch Colorectal Cancer Trial, mitigated local recurrence, and postoperative chemotherapy decreased the risk of distant metastases. Furthermore, the concept of radiosensitization had not yet been clearly proven in rectal cancer, because the Intergroup/NCCTG 86-47-51 trial did not show an improvement in pelvic control, despite its improvement in OS. Therefore, several trials have sought to address the need for chemotherapy during preoperative chemoradiation.

Two trials addressed this issue by comparing short-course radiation alone with standard preoperative chemoradiation. A Polish study and a study conducted by the Trans-Tasman Radiation Oncology Group (TROG) randomized 312 patients with clinically staged T3 low rectal cancer and 326 patients who had ultrasound- or MRI-staged rectal cancer, respectively, to either standard preoperative 50.4 Gy of chemoradiation with 5-FU and leucovorin or 25 Gy of radiation in 5 fractions without chemotherapy. Both studies showed no difference between the arms with respect to OS, DFS, local recurrence, or toxicity. ${ }^{21,22}$

Two trials also addressed the role of concurrent chemotherapy in the setting of conventionally fractionated radiation therapy. Fédération Francophone de Cancérologie Digestive (FFCD) 9203 randomized 733 preoperative patients with locally advanced rectal cancer to 45 Gy radiation alone or 45 Gy with concurrent chemotherapy with bolus 5-FU and leucovorin. ${ }^{23}$ Both groups received 4 cycles of adjuvant 5-FU/leucovorin after surgery. A significant decrease in local recurrence at 5 years was seen in the chemoradiation arm ( $8 \%$ vs. $16 \%)$, with no benefit seen in OS. EORTC 22921 was a $2 \times 2$ trial that randomized patients with T3 or T4 rectal cancer to preoperative chemoradiation with continuous-infusion 5-FU or radiation alone followed by either postoperative chemotherapy with 5-FU or no further treatment. ${ }^{24}$ No progression-free survival or OS benefit was seen in the group that received postoperative chemotherapy. However, local control was improved in all chemotherapy-containing arms, regardless of whether the chemotherapy was given concurrently or postoperatively.

The current standard of care in the United States is preoperative chemoradiation. However, this question is still being investigated and some centers offer short-course preoperative radiation without chemotherapy in patients unable to tolerate a full course of chemoradiation.

\section{Attempts to Improve on Bolus or Continuous-Infusion 5-FU and Radiation}

Advances in chemotherapeutics have led to multiple trials that attempt to improve the $\mathrm{pCR}$ rate of chemoradiation in rectal cancer. Capecitabine is an oral prodrug that undergoes conversion to 5-FU. Patients prescribed capecitabine no longer require connection to a pump for continuous infusion, which has the potential to significantly improve their quality of life during treatment. However, data comparing capecitabine with 5-FU are still early in development. In the NSABP R-04 trial, 1608 patients with locally advanced rectal cancer treated with preoperative radiation to 50.4 to 55.8 Gy were randomized to 1 of 4 different concurrent chemotherapies: continuous-infusion 5-FU; continuous-infusion 5-FU with oxaliplatin; capecitabine; or capecitabine and oxaliplatin. Preliminary data thus far show no difference in pCR, sphincter-sparing surgery, and surgical downstaging. ${ }^{25}$

Three randomized trials have sought to improve the efficacy of preoperative chemoradiation by adding oxaliplatin to standard regimens because of its established role in the postoperative setting for resected colon cancer. The Studio Terapia Adiuvante Retto (STAR) 01 trial randomized 747 patients to standard preoperative chemoradiation with continuous-infusion 5-FU alone followed by surgery and postoperative 5-FU-based chemotherapy or the same regimen with the addition of oxaliplatin. ${ }^{26}$ The ACCORD 12/0405-Prodige 2 trial randomized patients to preoperative radiation with capecitabine alone or with oxaliplatin. ${ }^{27}$ The NSABP R-04 trial also contained arms with oxaliplatin in addition to either capecitabine or 5-FU. ${ }^{25}$ All 3 trials showed no benefit in efficacy end points ( $\mathrm{pCR}$ rates for the STAR and ACCORD 12/0405-Prodige 2 trials, and locoregional recurrence rates for NSABP R-04), and significant increases in toxicity with the addition of oxaliplatin to chemoradiation. ${ }^{25-27}$

\section{Timing of Chemoradiation}

Little data exist on the optimal interval between preoperative chemoradiation and surgery. In 
randomized trials, surgery is typically recommended between 3 and 10 weeks after the completion of chemoradiation. $^{23,24}$ In trials that have used the short-course regimen of 25 Gy given in 5 days over a single week, surgery has been performed around 1 to 2 weeks after radiation. ${ }^{11,21,28}$ The French Lyons R90-01 trial used a nonstandard preoperative fractionation schedule (38 Gy in 13 fractions) followed by either a 2 -week or a 6 - to 8 -week interval to surgery. ${ }^{29}$ They showed the longer interval to surgery improved tumor downstaging without increasing surgical toxicities. A trend was also seen toward increased sphincter-preserving surgery in the long interval arm. In standard clinical practice, surgery is commonly performed between 4 and 10 weeks after the completion of chemoradiation.

\section{Evolution of Adjuvant Postoperative Chemotherapy Regimens}

The development of systemic chemotherapy regimens for adjuvant therapy in patients with locally advanced rectal cancer was borne out of initial success with 5-FU in colon cancer. ${ }^{30,31}$ NSABP R-01 randomized 555 patients with locally advanced rectal cancer to receive postoperative observation, 5 -FU/semustine/ vincristine $(\mathrm{MOF})$, or radiation alone to $46 \mathrm{~Gy}{ }^{4}$ This trial showed a benefit in OS and DFS in the chemotherapy group only. The radiation arm showed a decrease in locoregional recurrence from $25 \%$ to $16 \%$ without a change in DFS or OS. This helped solidify the role of postoperative chemotherapy in rectal cancer.

The use of 5-FU-based chemotherapy in the postoperative setting has been evaluated in the early adjuvant randomized trials and has consistently shown an improvement in OS. ${ }^{1,2}$ The relative benefit in risk reduction of metastatic disease was consistently approximately one-third, similar to the reduction seen in adjuvant studies in stage III rectal cancer.

Because of the similar benefits in colon and rectal cancer seen with chemotherapy, increased interest has been shown in the use of oxaliplatin in rectal cancer regimens, driven by DFS and OS benefits seen in adjuvant colon cancer studies. In the MOSAIC study, patients with stage II and III colon cancer were randomized to receive adjuvant 5-FU/ leucovorin with or without oxaliplatin. ${ }^{32}$ A significant improvement was seen in 3-year DFS (78.2\% vs. $72.9 \%$ ). Subsequent long-term follow-up shows that this benefit extends to OS. ${ }^{33}$ Extrapolating from these results, a combination of 5-FU, leucovorin, and oxaliplatin (FOLFOX) has become the standard of care for postoperative chemotherapy. A study led by ECOG attempting to evaluate FOLFOX in the adjuvant setting for patients with stage II and III rectal cancer was closed because of poor accrual, and the cooperative groups currently have no plans to evaluate the role of FOLFOX versus 5-FU/leucovorin in the adjuvant setting of rectal cancer. ${ }^{34}$

EORTC 22921 was a 2 × 2 trial that randomized patients with $\mathrm{T} 3$ or $\mathrm{T} 4$ rectal cancer to preoperative chemoradiation with continuous-infusion 5-FU or radiation alone followed by either postoperative chemotherapy with 5-FU or no further treatment. ${ }^{24}$ No PFS or OS benefit was seen in the entire group with the addition of chemotherapy. However, a benefit in both DFS and OS was seen in patients who were downstaged to T0-T2 at surgery. A systematic review of 4 trials that combined elements of modern treatment (TME surgery and preoperative chemoradiation) with or without postoperative 5-FU-based chemotherapy in patients treated with preoperative chemoradiation also found no benefit for adjuvant postoperative chemotherapy. ${ }^{35}$ This raises questions about current guideline recommendations and the need for new trials evaluating the efficacy of postoperative chemotherapy in the modern era and tailoring postoperative chemotherapy to patients at highest risk of systemic recurrence.

\section{Targeted Therapies in Rectal Cancer}

With the increasing availability of biologic agents active in colon and rectal cancer, integration of these agents into current standard of care is under investigation. Most studies have focused on the use of agents that target 1 of 2 pathways: vascular endothelial growth factor (VEGF) and epidermal growth factor receptor (EGFR). ${ }^{36}$

\section{VEGF Inhibition}

VEGF is a circulating glycoprotein involved in angiogenesis through interactions with the VEGF family of receptors. VEGF plays a role in both normal and pathologic blood vessel formation. The ability to recruit vessels is a critical event in tumor progression and can portend a negative prognosis and 
higher stage. ${ }^{37}$ Blocking tumor angiogenesis through VEGF inhibition became an attractive anticancer target. However, VEGF activation has far-reaching effects, including endothelial cell proliferation and migration, and effects on vascular permeability and vasodilation. Although initially thought to exert its effects through primarily antiangiogenic mechanisms, VEGF inhibition has been shown to have multiple effects on the tumor microenvironment and tumor cell type-specific effects. ${ }^{38}$

Bevacizumab is an anti-VEGF monoclonal antibody initially developed in colorectal cancer. Increased VEGF levels in colorectal cancers have been shown to predict poor outcomes..$^{39,40}$ Bevacizumab initially showed activity in a phase II trial in metastatic colorectal cancer. ${ }^{41}$ A follow-up phase III trial with 813 patients showed improved response rates and a benefit in median OS from 15.6 to 20.3 months $(P<.001) .{ }^{42}$ This led to FDA approval of bevacizumab used in a 5 -FU-based regimen as first-line treatment in metastatic colorectal cancer.

This activity in colorectal cancer led to interest in integrating bevacizumab in the adjuvant setting, particularly during the chemoradiation phase of treatment of rectal cancer. Willett et $\mathrm{al}^{43}$ performed phase I/II trials integrating bevacizumab into 5-FUbased chemoradiation regimens. An initial phase I study enrolled 32 patients with histologically confirmed rectal adenocarcinoma with $\mathrm{T} 3$ or $\mathrm{T} 4$ lesions based on endoscopic ultrasound or endorectal coil MRI. ${ }^{44}$ All patients received 4 cycles of therapy: bevacizumab ( 5 or $10 \mathrm{mg} / \mathrm{kg}$ ) on day 1; 5-FU infusion at $225 \mathrm{mg} / \mathrm{m}^{2}$ during cycles 2 through 4; and $5040 \mathrm{cGy}$ of radiation to the pelvis delivered in 28 fractions over 5.5 weeks followed by surgery. Most (30 of 32) patients received postoperative chemotherapy. The pCR rate was $16 \%$, actuarial 5-year local control and OS rates were $100 \%$, and $40 \%$ of patients experienced postoperative complications. Crane et $\mathrm{al}^{45}$ reported a pCR rate of $32 \%$ (8 of 25 patients) treated in a similar phase II trial combining capecitabine and bevacizumab with pelvic radiation. This trial reported 8 wound complications with 3 requiring surgical intervention. This high-rate of surgical complications merits consideration in design of larger trials.

Czito et $\mathrm{al}^{46}$ reported on a similar phase I dose-escalation trial using the combination of capecitabine, oxaliplatin, and bevacizumab with concurrent radiation in 11 patients with rectal adenocarcinoma. Nine patients had pathologic downstaging relative to initial clinical staging. Two patients had a pCR and 3 had microscopic disease only. Kennecke et $\mathrm{al}^{47}$ reported a pCR rate of $23.7 \%$ in a similar phase II trial using capecitabine, oxaliplatin, and bevacizumab with radiation.

\section{EGFR Inhibition}

EGFR is a member of a family of tyrosine kinases that includes HER2. Ligand binding of the extracellular domain leads to a cascade of events that relays growth and division signals to the nucleus of the cell. Downstream pathways involved in this relay include the ras-raf mitogen-activated protein kinase (MAPK) pathway and the phosphoinositide 3-kinase (PI3K)-AKT-mTOR pathway. Expression or upregulation of EGFR occurs in $60 \%$ to $80 \%$ of patients with metastatic colorectal cancer. ${ }^{48,49}$

Two types of EGFR-targeted therapies have been developed and studied in rectal cancer: monoclonal antibodies that target the extracellular domain (eg, cetuximab and panitumumab) and small molecule tyrosine kinase inhibitors (eg, gefitinib and erlotinib).

Gefitinib has not gained widespread use in rectal cancer because of its high toxicity rates. Valentini et $\mathrm{al}^{50}$ treated 41 patients with clinical T3/4 or nodepositive rectal cancer with continuous-infusion 5 -FU and gefitinib with pelvic radiation, and reported a pCR rate of $30 \%$. However, greater than $61 \%$ of patients required dose reductions for grade 3 or higher toxicity. Panitumumab has faced similar difficulties in its use in rectal cancer. Pinto et $\mathrm{al}^{51}$ reported results of a phase II trial in which 60 patients with $\mathrm{T} 3, \mathrm{~N}+$ or $\mathrm{T} 4, \mathrm{~N}-/+$ rectal cancer were treated with panitumumab combined with oxaliplatin, 5-FU, and pelvic radiation followed by surgery. They reported a pCR rate of $21.1 \%$. However, grade 3 toxicity was high and consisted primarily of diarrhea (38.9\%) and cutaneous reactions $(18.6 \%)$, with 1 treatmentrelated death from refractory diarrhea.

Early studies with cetuximab-based chemoradiation in head and neck cancers showed both survival and locoregional control benefit. ${ }^{52}$ Multiple phase I/II studies combining chemoradiation and cetuximab in locally advanced rectal cancer have been disappointing, with reported pCR rates ranging from $5 \%$ to $12 \% .{ }^{53-59}$ This lack of response may be from common mutations in rectal cancer that override upstream EGFR inhibition. Multiple studies have shown that 
response to cetuximab and panitumumab occur only in patients without activating mutations in the $\mathrm{k}$-ras proto-oncogene. ${ }^{60-63}$ Ongoing investigations in the use of cetuximab in rectal cancer integrate $\mathrm{k}$-ras mutation status in treatment algorithms.

\section{Combination VEGF/EGFR Therapies}

Two large randomized trials, the Panitumumab Advanced Colorectal Cancer Evaluation (PACCE) and CAIRO2, showed no benefit and increased toxicity in patients with metastatic colorectal treated with 5-FU, oxaliplatin or irinotecan, and leucovorin randomized to combination EGFR and VEGF-targeted therapies compared with VEGFtargeted therapies alone. ${ }^{64,65}$

In a phase I/II study of bevacizumab and erlotinib in combination with 5-FU-based chemoradiation, Blaszkowsky et $\mathrm{al}^{66}$ reported a $47 \%$ pCR rate with acceptable toxicity. In a similar phase I study, Das et $\mathrm{al}^{67}$ reported a $44 \% \mathrm{pCR}$ rate with the combination of bevacizumab, erlotinib, capecitabine, and radiation.

The role of targeted therapies in the treatment of patients with rectal cancer is continually evolving. New drugs, including those targeting BRAF and the $\mathrm{PI} 3 \mathrm{~K} / \mathrm{AKT} / \mathrm{mTOR}$ pathway, are being investigated and their activity in rectal cancer has yet to be defined. As targeted therapies evolve, the use of combination treatment retains promise as a means of preventing salvage pathway activation. Currently, the use of EGFR and VEGF inhibitors in adjuvant therapy for rectal cancer should be restricted to the setting of clinical trials.

\section{Conclusions}

Although great strides have been made over the past 2 decades in the treatment of locally advanced rectal cancer, many areas of open investigation remain. The tremendous improvement in local control through use of TME has focused research efforts on better control of systemic disease. Although early trial results have been disappointing, targeted therapeutics remain promising as discoveries in salvage pathways and new methods of combined therapies develop. A better understanding of the molecular heterogeneity of rectal cancers should help guide selection of appropriate treatments for patients.

\section{References}

1. Radiation therapy and fluorouracil with or without semustine for the treatment of patients with surgical adjuvant adenocarcinoma of the rectum. Gastrointestinal Tumor Study Group. J Clin Oncol 1992;10:549-557.

2. Fisher B, Wolmark N, Rockette $H$, et al. Postoperative adjuvant chemotherapy or radiation therapy for rectal cancer: results from NSABP protocol R-01. J Natl Cancer Inst 1988;80:21-29.

3. Krook JE, Moertel CG, Gunderson LL, et al. Effective surgical adjuvant therapy for high-risk rectal carcinoma. N Engl J Med 1991;324:709-715.

4. O'Connell MJ, Martenson JA, Wieand HS, et al. Improving adjuvant therapy for rectal cancer by combining protractedinfusion fluorouracil with radiation therapy after curative surgery. N Engl J Med 1994;331:502-507.

5. Wolmark N, Wieand HS, Hyams DM, et al. Randomized trial of postoperative adjuvant chemotherapy with or without radiotherapy for carcinoma of the rectum: National Surgical Adjuvant Breast and Bowel Project protocol R-02. J Natl Cancer Inst 2000;92:388396.

6. Heald RJ, Husband EM, Ryall RD. The mesorectum in rectal cancer surgery-the clue to pelvic recurrence? Br J Surg 198269:613-616.

7. Heald RJ, Ryall RD. Recurrence and survival after total mesorectal excision for rectal cancer. Lancet 1986;1:1479-1482.

8. MacFarlane JK, Ryall RD, Heald RJ. Mesorectal excision for rectal cancer. Lancet 1993;341:457-460.

9. Enker WE, Thaler HT, Cranor ML, et al. Total mesorectal excision in the operative treatment of carcinoma of the rectum. J Am Coll Surg 1995;181:335-346.

10. Havenga K, Enker WE, Norstein J, et al. Improved survival and local control after total mesorectal excision or D3 lymphadenectomy in the treatment of primary rectal cancer: an international analysis of 1411 patients. Eur J Surg Oncol 1999;25:368-374.

11. Kapiteijn E, Marijnen CA, Nagtegaal ID, et al. Preoperative radiotherapy combined with total mesorectal excision for resectable rectal cancer. N Engl J Med 2001;345:638-646.

12. van Gijn W, Marijnen CA, Nagtegaal ID, et al. Preoperative radiotherapy combined with total mesorectal excision for resectable rectal cancer: 12-year follow-up of the multicentre, randomised controlled TME trial. Lancet Oncol 2011;12:575-582.

13. Koehler PR, Feldberg MA, van Waes PF. Preoperative staging of rectal cancer with computerized tomography. Accuracy, efficacy, and effect on patient management. Cancer 1984;54:512-516.

14. Beynon J, Mortensen NJ, Foy DM, et al. Pre-operative assessment of local invasion in rectal cancer: digital examination, endoluminal sonography or computed tomography? Br J Surg 1986;73:10151017.

15. Rifkin MD, Ehrlich SM, Marks G. Staging of rectal carcinoma: prospective comparison of endorectal US and CT. Radiology 1989;170:319-322.

16. Rifkin MD, Wechsler RJ. A comparison of computed tomography and endorectal ultrasound in staging rectal cancer. Int J Colorectal Dis 1986;1:219-223.

17. de Lange EE, Fechner RE, Edge SB, et al. Preoperative staging of rectal carcinoma with MR imaging: surgical and histopathologic correlation. Radiology 1990;176:623-628.

18. Roh MS, Colangelo LH, O'Connell MJ, et al. Preoperative multimodality therapy improves disease-free survival in patients with carcinoma of the rectum: NSABP R-03. J Clin Oncol 2009;27:5124-5130. 
19. Sauer R, Liersch $T$, Merkel $S$, et al. Preoperative versus postoperative chemoradiotherapy for locally advanced rectal cancer: results of the German CAO/ARO/AIO-94 randomized phase III trial after a median follow-up of 11 years [abstract]. J Clin Oncol 2012;29(Suppl):Abstract 3516.

20. Sauer R, Becker H, Hohenberger W, et al. Preoperative versus postoperative chemoradiotherapy for rectal cancer. N Engl J Med 2004;351:1731-1740.

21. Bujko K, Nowacki MP, Nasierowska-Guttmejer A, et al. Longterm results of a randomized trial comparing preoperative shortcourse radiotherapy with preoperative conventionally fractionated chemoradiation for rectal cancer. Br J Surg 2006;93:1215-1223.

22. Ngan S, Fisher R, Goldstein D, et al. A randomized trial comparing local recurrence (LR) rates between short-course (SC) and longcourse (LC) preoperative radiotherapy (RT) for clinical T3 rectal cancer: an intergroup trial (TROG, AGITG, CSSANZ, RACS) [abstract]. J Clin Oncol 2010(Suppl):Abstract 3509.

23. Gerard JP, Conroy T, Bonnetain F, et al. Preoperative radiotherapy with or without concurrent fluorouracil and leucovorin in T3-4 rectal cancers: results of FFCD 9203. J Clin Oncol 2006;24:46204625.

24. Collette L, Bosset JF, den Dulk M, et al. Patients with curative resection of cT3-4 rectal cancer after preoperative radiotherapy or radiochemotherapy: does anybody benefit from adjuvant fluorouracil-based chemotherapy? A trial of the European Organisation for Research and Treatment of Cancer Radiation Oncology Group. J Clin Oncol 2007;25:4379-4386.

25. Roh MS, Yothers GA, O'Connell MJ, et al. The impact of capecitabine and oxaliplatin in the preoperative multimodality treatment in patients with carcinoma of the rectum: NSABP R-04 [abstract]. J Clin Oncol 2011;29(Suppl):Abstract 3503.

26. Aschele $\mathrm{C}$, Cionini L, Lonardi $\mathrm{S}$, et al. Primary tumor response to preoperative chemoradiation with or without oxaliplatin in locally advanced rectal cancer: pathologic results of the STAR-01 randomized phase III trial. J Clin Oncol 2011;29:2773-2780.

27. Gerard JP, Azria D, Gourgou-Bourgade S, et al. Comparison of two neoadjuvant chemoradiotherapy regimens for locally advanced rectal cancer: results of the phase III trial ACCORD 12/0405-Prodige 2. J Clin Oncol 2010;28:1638-1644.

28. Sebag-Montefiore D, Stephens RJ, Steele R, et al. Preoperative radiotherapy versus selective postoperative chemoradiotherapy in patients with rectal cancer (MRC CR07 and NCIC-CTG C016): a multicentre, randomised trial. Lancet 2009;373:811-820.

29. Francois $\mathrm{Y}, \mathrm{Nemoz} C J$, Baulieux J, et al. Influence of the interval between preoperative radiation therapy and surgery on downstaging and on the rate of sphincter-sparing surgery for rectal cancer: the Lyon R90-01 randomized trial. J Clin Oncol 1999;17:2396.

30. Moertel CG, Fleming TR, Macdonald JS, et al. Levamisole and fluorouracil for adjuvant therapy of resected colon carcinoma. N Engl J Med 1990;322:352-358.

31. Haller DG, Catalano PJ, Macdonald JS, et al. Phase III study of fluorouracil, leucovorin, and levamisole in high-risk stage II and III colon cancer: final report of Intergroup 0089. J Clin Oncol 2005;23:8671-8678

32. Andre $\mathrm{T}$, Boni C, Mounedji-Boudiaf L, et al. Oxaliplatin, fluorouracil, and leucovorin as adjuvant treatment for colon cancer. N Engl J Med 2004;350:2343-2351.

33. Andre $\mathrm{T}$, Boni $\mathrm{C}$, Navarro $\mathrm{M}$, et al. Improved overall survival with oxaliplatin, fluorouracil, and leucovorin as adjuvant treatment in stage II or III colon cancer in the MOSAIC trial. J Clin Oncol 2009;27:3109-3116.
34. Benson AB, Catalano PJ, Meropol NJ, et al. ECOG E3201 Intergroup randomized phase III study of postoperative irinotecan, 5- fluorouracil (FU), leucovorin (LV) (FOLFIRI) vs oxaliplatin, FU/LV (FOLFOX) vs FU/LV for patients (pts) with stage II/ III rectal cancer receiving either pre or postoperative radiation (RT)/ FU [abstract]. J Clin Oncol 2006;24(Suppl):Abstract 3526.

35. Bujko K, Glynne-Jones R, Bujko M. Does adjuvant fluoropyrimidine-based chemotherapy provide a benefit for patient with resected rectal cancer who have already received neoadjuvant radiochemotherapy? A systematic review of randomised trials. Ann Oncol 2010;21:1743-1750.

36. Wadlow RC, Ryan DP. The role of targeted agents in preoperative chemoradiation for rectal cancer. Cancer 2010;116:3537-3548.

37. Hicklin DJ, Ellis LM. Role of the vascular endothelial growth factor pathway in tumor growth and angiogenesis. J Clin Oncol 2005;23:1011-1027.

38. Ellis LM, Hicklin DJ. VEGF-targeted therapy: mechanisms of antitumour activity. Nat Rev Cancer 2008;8:579-591.

39. Choi HJ, Hyun MS, Jung GJ, et al. Tumor angiogenesis as a prognostic predictor in colorectal carcinoma with special reference to mode of metastasis and recurrence. Oncology 1998;55:575-581.

40. Frank RE, Saclarides TJ, Leurgans $S$, et al. Tumor angiogenesis as a predictor of recurrence and survival in patients with node-negative colon cancer. Ann Surg 1995;222:695-699.

41. Kabbinavar F, Hurwitz HI, Fehrenbacher L, et al. Phase II, randomized trial comparing bevacizumab plus fluorouracil (FU)/ leucovorin (LV) with FU/LV alone in patients with metastatic colorectal cancer. J Clin Oncol 2003;21:60-65.

42. Hurwitz H, Fehrenbacher L, Novotny W, et al. Bevacizumab plus irinotecan, fluorouracil, and leucovorin for metastatic colorectal cancer. N Engl J Med 2004;350:2335-2342.

43. Willett CG, Boucher Y, Duda DG, et al. Surrogate markers for antiangiogenic therapy and dose-limiting toxicities for bevacizumab with radiation and chemotherapy: continued experience of a phase I trial in rectal cancer patients. J Clin Oncol 2005;23:8136-8139.

44. Willett CG, Duda DG, di Tomaso E, et al. Efficacy, safety, and biomarkers of neoadjuvant bevacizumab, radiation therapy, and fluorouracil in rectal cancer: a multidisciplinary phase II study. J Clin Oncol 2009;27:3020-3026.

45. Crane $\mathrm{CH}$, Eng C, Feig BW, et al. Phase II trial of neoadjuvant bevacizumab, capecitabine, and radiotherapy for locally advanced rectal cancer. Int J Radiat Oncol Biol Phys 2010;76:824-830.

46. Czito BG, Bendell JC, Willett CG, et al. Bevacizumab, oxaliplatin, and capecitabine with radiation therapy in rectal cancer: phase I trial results. Int J Radiat Oncol Biol Phys 2007;68:472-478.

47. Kennecke H, Berry S, Wong R, et al. Pre-operative bevacizumab, capecitabine, oxaliplatin and radiation among patients with locally advanced or low rectal cancer: a phase II trial. Eur J Cancer 2012;48:37-45.

48. Messa C, Russo F, Caruso MG, et al. EGF, TGF-alpha, and EGF-R in human colorectal adenocarcinoma. Acta Oncol 1998;37:285289.

49. Porebska I, Harlozinska A, Bojarowski T. Expression of the tyrosine kinase activity growth factor receptors (EGFR, ERB B2, ERB B3) in colorectal adenocarcinomas and adenomas. Tumour Biol 2000;21:105-115.

50. Valentini V, De Paoli A, Gambacorta MA, et al. Infusional 5-fluorouracil and ZD1839 (Gefitinib-Iressa) in combination with preoperative radiotherapy in patients with locally advanced rectal cancer: a phase I and II trial (1839IL/0092). Int J Radiat Oncol Biol Phys 2008;72:644-649. 
51. Pinto C, Di Fabio F, Maiello E, et al. Phase II study of panitumumab, oxaliplatin, 5-fluorouracil, and concurrent radiotherapy as preoperative treatment in high-risk locally advanced rectal cancer patients (StarPan/STAR-02 Study). Ann Oncol 2011;22:24242430.

52. Bonner JA, Harari PM, Giralt J, et al. Radiotherapy plus cetuximab for squamous-cell carcinoma of the head and neck. N Engl J Med 2006;354:567-578.

53. Bertolini F, Chiara S, Bengala C, et al. Neoadjuvant treatment with single-agent cetuximab followed by 5 -FU, cetuximab, and pelvic radiotherapy: a phase II study in locally advanced rectal cancer. Int J Radiat Oncol Biol Phys 2009;73:466-472.

54. Dewdney A, Cunningham D, Tabernero J, et al. Multicenter randomized phase II clinical trial comparing neoadjuvant oxaliplatin, capecitabine, and preoperative radiotherapy with or without cetuximab followed by total mesorectal excision in patients with high-risk rectal cancer (EXPERT-C). J Clin Oncol 2012;30:1620-1627.

55. Hofheinz RD, Horisberger K, Woernle C, et al. Phase I trial of cetuximab in combination with capecitabine, weekly irinotecan, and radiotherapy as neoadjuvant therapy for rectal cancer. Int J Radiat Oncol Biol Phys 2006;66:1384-1390.

56. Horisberger K, Treschl A, Mai S, et al. Cetuximab in combination with capecitabine, irinotecan, and radiotherapy for patients with locally advanced rectal cancer: results of a phase II MARGIT trial. Int J Radiat Oncol Biol Phys 2009;74:1487-1493.

57. Machiels JP, Sempoux C, Scalliet P, et al. Phase I/II study of preoperative cetuximab, capecitabine, and external beam radiotherapy in patients with rectal cancer. Ann Oncol 2007;18:738-744.

58. Rodel C, Arnold D, Hipp M, et al. Phase I-II trial of cetuximab, capecitabine, oxaliplatin, and radiotherapy as preoperative treatment in rectal cancer. Int J Radiat Oncol Biol Phys 2008;70:1081-1086.
59. Chung KY, Minsky B, Schrag D, et al. Phase I trial of preoperative cetuximab with concurrent continuous infusion 5-fluorouracil and pelvic radiation in patients with local-regionally advanced rectal cancer [abstract]. J Clin Oncol 2006;24(Suppl):Abstract 3560.

60. Amado RG, Wolf M, Peeters M, et al. Wild-type KRAS is required for panitumumab efficacy in patients with metastatic colorectal cancer. J Clin Oncol 2008;26:1626-1634.

61. Bokemeyer C, Bondarenko I, Hartmann JT, et al. Efficacy according to biomarker status of cetuximab plus FOLFOX-4 as first-line treatment for metastatic colorectal cancer: the OPUS study. Ann Oncol 2011;22:1535-1546.

62. Karapetis CS, Khambata-Ford S, Jonker DJ, et al. K-ras mutations and benefit from cetuximab in advanced colorectal cancer. N Engl J Med 2008;359:1757-1765.

63. Van Cutsem E, Kohne $\mathrm{CH}$, Hitre E, et al. Cetuximab and chemotherapy as initial treatment for metastatic colorectal cancer. N Engl J Med 2009;360:1408-1417.

64. Hecht JR, Mitchell E, Chidiac T, et al. A randomized phase IIIB trial of chemotherapy, bevacizumab, and panitumumab compared with chemotherapy and bevacizumab alone for metastatic colorectal cancer. J Clin Oncol 2009;27:672-680.

65. Tol J, Koopman M, Cats A, et al. Chemotherapy, bevacizumab, and cetuximab in metastatic colorectal cancer. N Engl J Med 2009;360:563-572.

66. Blaszkowsky LS, Hong TS, Zhu AX, et al. A phase I/II study of bevacizumab (beva), erlotinib (erl), and 5-fluorouracil (5FU) with concurrent external beam radiation therapy (RT) in locally advanced rectal cancer (LARC) [abstract]. J Clin Oncol 2009;27(Suppl):Abstract 4106

67. Das $\mathrm{P}$, Eng C, Rodriguez-Bigas MA, et al. Preoperative radiation therapy with concurrent capecitabine, bevacizumab, and erlotinib for rectal adenocarcinoma: a phase I trial [abstract]. J Clin Oncol 2012;30(Suppl):Abstract 544.

\section{Instructions for Completion}

To participate in this journal CE activity: 1) review the learning objectives and author disclosures; 2) study the education content; 3) take the post-test with a $70 \%$ minimum passing score and complete the evaluation at http://education.nccn. org/node/6277; and 4) view/print certificate. After reading the article, you should be able to answer the following multiple- choice questions. Credit cannot be obtained for tests completed on paper. You must be a registered user on NCCN.org. If you are not registered on NCCN.org, click on "New Member? Sign up here" link on the left hand side of the Web site to register. Only one answer is correct for each question. Once you successfully answer all post-test questions you will be able to view and/or print your certificate. Software requirements: Internet

\section{Post-Test Questions}

1. True or False: In the United States, the current standard of care for patients with locally advanced rectal cancer is neoadjuvant chemoradiation followed by surgery and adjuvant chemotherapy.

2. Which of the following is NOT a target pathway for targeted therapies in rectal cancer?

a. Vascular endothelial growth factor (VEGF)

b. Anaplastic lymphoma kinase (ALK)

c. Epidermal growth factor receptor (EGFR)
3. True or False: The use of 5-FUbased chemotherapy in patients with rectal cancer in the postoperative setting has been evaluated in early adjuvant randomized trials and has consistently shown an improvement in overall survival.

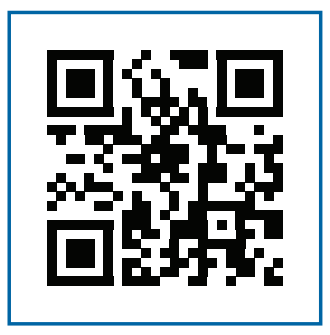

